TRANSACTIONS OF THE

AMERICAN MATHEMATICAL SOCIETY

Volume 348, Number 11, November 1996

\title{
THE BOUNDARY OF ITERATES IN EUCLIDEAN GROWTH MODELS
}

\author{
JANKO GRAVNER
}

\begin{abstract}
This paper defines a general Euclidean growth model via a translation invariant, monotone and local transformation on Borel subsets of $\mathbf{R}^{2}$. The main result gives a geometric condition for the boundary curvature of the iterates to go to 0 . Consequences include estimates for the speed of convergence to the asymptotic shape, and a result about survival of Euclidean deterministic forest fires.
\end{abstract}

\section{INTRODUCTION}

Let $\mathcal{B}$ be the $\sigma$-algebra of Borel subsets of $\mathbf{R}^{2}$. We call a map $\mathcal{T}: \mathcal{B} \rightarrow \mathcal{B}$ a twodimensional growth transformation $(G T)$ if it satisfies the following four conditions.

(1) It is translation invariant: for all $A \in \mathcal{B}$ and $x \in \mathbf{R}^{2}, \mathcal{T}(x+A)=x+\mathcal{T}(A)$.

(2) It is monotone: for every $A, B \in \mathcal{B}, A \subset B$ implies $\mathcal{T}(A) \subset \mathcal{T}(B)$.

(3) It is local: there exists a constant $R>0$ so that for every $A \in \mathcal{B}, \mathcal{T}(A)=$ $\left\{x \in \mathbf{R}^{2}: x \in \mathcal{T}\left(A \cap B_{2}(x, R)\right)\right\}$. (We use the standard notation for Euclidean balls: $B_{p}(x, R)=\left\{y \in \mathbf{R}^{2}:\|x-y\|_{p} \leq R\right\}$.)

(4) $\mathcal{T}(\emptyset)=\emptyset, \mathcal{T}\left(\mathbf{R}^{2}\right)=\mathbf{R}^{2}$.

The iterates of a GT, given by $\mathcal{T}^{n}\left(A_{0}\right)$ for a fixed initial set $A_{0}$, are called growth dynamics.

Assumption (4) avoids the trivial cases when $\mathcal{T}(\emptyset)=\mathbf{R}^{2}$ or $\mathcal{T}\left(\mathbf{R}^{2}\right)=\emptyset$ and, together with (3), assures that $\mathcal{T}(A)$ is bounded for every bounded $A$. In this connection, a neighborhood for $\mathcal{T}$ is any compact set $\mathcal{N}$ such that for every $x \in \mathbf{R}^{2}$ and $A \in \mathcal{B}, x \in \mathcal{T}(A) \Leftrightarrow x \in \mathcal{T}(A \cap(x+\mathcal{N}))$.

Before introducing several examples, we remark that the set of GT's is closed with respect to finite unions, intersections and convex combinations. More formally, let $\mathcal{T}_{1}, \mathcal{T}_{2}, \ldots$ be a finite collection of GT's, and $\lambda_{1}, \lambda_{2}, \cdots \geq 0$ with $\sum_{n} \lambda_{n}=1$. Then the growth transformations $\bigcup_{n} \mathcal{T}_{n}, \bigcap_{n} \mathcal{T}_{n}$ and $\sum_{n} \lambda_{n} \mathcal{T}_{n}$ are defined by

$$
\begin{gathered}
\left(\bigcup_{n} \mathcal{T}_{n}\right)(A)=\bigcup_{n} \mathcal{T}_{n}(A), \quad\left(\bigcap_{n} \mathcal{T}_{n}\right)(A)=\bigcap_{n} \mathcal{T}_{n}(A), \\
\left(\sum_{n} \lambda_{n} \mathcal{T}_{n}\right)(A)=\sum_{n} \lambda_{n} \mathcal{T}_{n}(A) .
\end{gathered}
$$

Received by the editors July 14, 1995.

1991 Mathematics Subject Classification. Primary 52A10; Secondary 52A99, 60K35.

Key words and phrases. Growth dynamics, curvature, deterministic forest fire.

This research was partially supported by the research grant J1-6157-0101-94 from the Republic of Slovenia's Ministry of Science. 
The basic example we have in mind is threshold growth dynamics, with parameters $\mu$, a measure on $\mathbf{R}^{2}$ with compact support, and threshold $\theta \geq 0$, which is given by $\mathcal{T}(A)=\left\{x \in \mathbf{R}^{d}: \mu(A-x) \geq \theta\right\}$. Various versions of threshold growth dynamics were introduced and analyzed in [GG1], [GG2] and [GG3], as natural models arising from the theories of excitable media, crystallization and percolation. Readers are referred to [DG], [FGG], [GG1] and [Gri], which help motivate threshold growth dynamics and provide many references. A flashy on-line introduction to the mathematical theory of excitable media and other aspects of deterministic and random cellular phenomena can be found at D. Griffeath's home page (http://math.wisc.edu/ ${ }^{\sim}$ griffeat/kitchen.html). Actually, the dynamics in the references just cited are formulated slightly differently, as $\overline{\mathcal{T}}(A)=A \cup \mathcal{T}(A)$. However, as soon as the initial set $A_{0}$ is large enough so that $A_{0} \subset \mathcal{T}\left(A_{0}\right)$, then $\mathcal{T}^{n}\left(A_{0}\right)=\overline{\mathcal{T}}^{n}\left(A_{0}\right)$, for every $n \geq 0$. For questions addressed in this paper, the difference between $\mathcal{T}$ and $\overline{\mathcal{T}}$ is therefore inessential.

Recent work of P. E. Souganidis and G. E. Pires ([Pir]) provides an alternative approach to the analysis of threshold growth in [GG1], via viscosity solutions of certain geometric PDE. However, these analytic methods fail to yield any information about rates of convergence and similar issues addressed here. In fact, the curvature estimates of the type developed in this paper may well be necessary for understanding how far the rescaled growth dynamics is from its continuous-time approximation.

Growth dynamics are additive if $\mathcal{T}(A \cup B)=\mathcal{T}(A) \cup \mathcal{T}(B)$ for every $A, B \in \mathcal{B}$. Although there are other examples (we leave it as a puzzle for the reader to find one), we will call Additive Dynamics only those defined by $\mathcal{T}(A)=A+\mathcal{N}$ for a fixed compact set $\mathcal{N}$. Additive Dynamics constitute virtually the only fully analyzed case of random growth (see [Dur], [NP]).

Inclusion Dynamics have two compact sets $\mathcal{M}, \mathcal{N} \in \mathcal{B}$ as parameters, and are defined by $\mathcal{T}(A)=\{x: y+\mathcal{M} \subset A \cap(x+\mathcal{N})$ for some $y\}$. Similarly, Diameter Dynamics are, for some compact $\mathcal{N} \in \mathcal{B}$ and $\theta \geq 0$, given by

$$
\mathcal{T}(A)=\{x: \operatorname{diam}(A \cap(x+\mathcal{N})) \geq \theta\} .
$$

We call the dynamics convex if $\mathcal{T}$ maps closed convex sets into closed convex sets. Not surprisingly, such a property greatly facilitates understanding of the boundary behavior when the initial set is convex. We will discuss this, and other preliminaries, in greater detail in Section 2.

One of the basic features of growth dynamics is the existence of an asymptotic shape. To formulate the result, we say that an initial set $A_{0}$ generates regular growth if for every compact set $F \subset \mathbf{R}^{2}$ there exists a large enough $n$ so that $F \subset \mathcal{T}^{n}\left(A_{0}\right)$. What exactly may happen if a bounded $A_{0}$ does not generate regular growth is not well understood (see [GG1] and [GG2] for some discussion on this point), although T. Bohman's remarkable recent argument ([Boh]) proves that the box-neighborhood discrete threshold growth dynamics started from a finite set either generates regular growth or stops. An important consequence of regularity is stated in the following theorem, which holds with a proof that easily generalizes the demonstration of Theorem 1 in [GG1].

Theorem 1.1. Let $\mathcal{T}$ be a growth transformation. If a bounded set $A_{0} \in \mathcal{B}$ generates regular growth, then there exists a closed convex bounded set $L$ such that $n^{-1} \mathcal{T}^{n}\left(A_{0}\right) \rightarrow L$. This convergence holds with respect to the Hausdorff distance $d_{H}$. 
In fact, $L$ can be identified explicitly in terms of half-spaces velocities. Note that any growth transformation $\mathcal{T}$ must translate half-planes: if $u \in S^{1}$ is a twodimensional unit vector and $H_{u}^{-}=\left\{x \in \mathbf{R}^{2}:\langle x, u\rangle \leq 0\right\}$, then there is a $w(u) \in \mathbf{R}$ so that $\mathcal{T}\left(H_{u}^{-}\right)$is either $H_{u}^{-}+w(u) u$ or $H_{u}^{-}+(-\infty, w(u)) u$. Now, define the set $K_{1 / w}=\bigcup_{u}[0,1 / w(u)] u$ and let

$$
L=K_{1 / w}^{*}=\left\{y:\langle x, y\rangle \leq 1 \text { for every } x \in K_{1 / w}\right\} .
$$

Once $L$ is defined by (1.1), it is possible to see that the theorem, as stated, is valid in any dimension, and under less restrictive conditions ([GG1] and [Gra] include details on a more general statement). We call the dynamics supercritical if there exists a bounded initial set which generates regular growth. Since it is easy to check that $w: S^{1} \rightarrow \mathbf{R}$ is continuous for any growth dynamics, supercriticality is equivalent to $w>0$ on $S^{1}$ (see [GG1]).

The intuition behind Theorem 1.1 and (1.1) is that the boundary of $\mathcal{T}^{n}(A)$ is essentially flat when $n$ is very large. This, as we will see momentarily, is not generally true, although it does provide the correct answer ([GG1]). To quantify the flatness of a closed convex set $B$, we say that $\nu \in S^{1}$ is a normal at $x \in \partial B$ if $B \subset x+H_{\nu}^{-}$and define, for an $a>0$,

$$
\begin{gathered}
m_{a}(B)=\sup \{\lambda>0: \text { for every } x \in \partial B \text { and every normal } \nu \text { at } x \\
\left.B_{2}(x-\lambda \nu, a) \cap\left(x-\lambda \nu+H_{\nu}^{-}\right) \subset B\right\} .
\end{gathered}
$$

If $K_{1 / w}$ is not convex, then $m_{a}\left(\mathcal{T}^{n}\left(A_{0}\right)\right)$ can never go to 0 ; in fact, even more is true.

Theorem 1.2. Assume the growth dynamics given by $\mathcal{T}$ are supercritical and convex. If $K_{1 / w}$ is not convex, then for every a $>0$ there exists an $\epsilon>0$ so that for every closed convex initial set $A_{0}, m_{a}\left(\mathcal{T}^{n}\left(A_{0}\right)\right)>\epsilon$ infinitely many times.

Proof. Without loss of generality, we can assume that $a$ is larger than $2 R$ (see (2.2)-(2.4)). Choose a $u \in S^{1}$ such that $w(u)^{-1} u \notin \partial\left(\operatorname{co}\left(K_{1 / w}\right)\right)$. (As usual, co(.) denotes the convex hull of a set.) Then there exists an $\epsilon>0$ so that $(w(u)-2 \epsilon)^{-1} u \in$ $\operatorname{co}\left(K_{1 / w}\right)$, and therefore $L \subset(w(u)-2 \epsilon) u+H_{u}^{-}$.

If $m_{a}\left(\mathcal{T}^{n}\left(A_{0}\right)\right) \leq \epsilon$ for all but finitely many $n$, then we can assume this holds for every $n$. However, then $\mathcal{T}^{n}\left(A_{0}\right)$ includes a point $x_{n} \notin n(w(u)-\epsilon) u+H_{u}^{-}$, and thus $n^{-1} \mathcal{T}^{n}\left(A_{0}\right)$ cannot converge to $L$, contradicting Theorem 1.1.

On the other hand, the following result holds, as we shall see in Section 3.

Theorem 1.3. Assume that the growth dynamics given by $\mathcal{T}$ are supercritical and convex, and that $K_{1 / w}$ is strictly convex. Fix an $a>0$.

(1) For every $\epsilon>0$ there exists a $\delta>0$ so that a convex initial set $A_{0}$ with $\mathcal{C}^{2}$ boundary and curvature $<\delta$ has $m_{a}\left(\mathcal{T}^{n}\left(A_{0}\right)\right)<\epsilon$ for every $n$.

(2) For every convex initial set $A_{0}$ that generates regular growth, $m_{a}\left(\mathcal{T}^{n}\left(A_{0}\right)\right) \rightarrow$ 0 as $n \rightarrow \infty$.

The boundary case about which we know nothing is when $K_{1 / w}$ is convex but not strictly convex. As examples of Additive Dynamics show (see Section 3), (2) above cannot generally hold in that case. Although it seems (1) might be true, our present techniques do not resolve the issue.

One reason for our interest in Theorem 1.3 is that the estimates in its proof yield information about the speed of convergence in Theorem 1.1. 
Theorem 1.4. Assume that the growth dynamics given by $\mathcal{T}$ are supercritical and convex, and that $K_{1 / w}$ is strictly convex and $\mathcal{C}^{2}$. Fix a convex initial set $A_{0}$ that generates regular growth, and such that $A_{0} \subset \mathcal{T}\left(A_{0}\right)$. Then there exist a constant $C$ so that $(n-C \log n) L \subset \mathcal{T}^{n}\left(A_{0}\right)$ for all sufficiently large $n$.

The proof of the upper bound in Theorem 1.1 (see [GG1]) shows that, under the above assumptions, $\mathcal{T}^{n}\left(A_{0}\right) \subset\left(n+C_{1}\right) L$ for some constant $C_{1}$. This, together with Theorem 1.4 gives more precise information about the behavior of iterates. For example, it follows immediately (see Lemma 2.7) that $m_{1}\left(\mathcal{T}^{n}\left(A_{0}\right)\right) \leq C_{2} \sqrt{\log n / n}$. We will show that in many instances $\mathcal{T}^{n}\left(A_{0}\right) \subset\left(n-C_{3} \log n\right) L$ for some constant $C_{3}>0$, in which case $\mathcal{T}^{n}\left(A_{0}\right)$ is of the order $\log n$ away from $n L$. In contrast, stochastic fluctuations in random growth models typically cause the distance between the growing set and $n L$ to obey a power law (see $[\mathrm{NP}]$ and references contained therein; note that strict convexity also plays an important role in the arguments of $[\mathrm{NP}])$.

However, our main motivation for Theorem 1.3 is the issue of survival of rings ([FGG], [GG1]). In [GG1], we introduced a simple Euclidean model called the deterministic forest fire (DFF) with the idea in mind that every occupied point can contribute to the growth during only once before it "burns away." At each time $n$, the state of the system is described by two Borel subsets of $\mathbf{R}^{2}, E_{n}$ and $F_{n}$. Then

$$
F_{n+1}=\mathcal{T}\left(F_{n}\right) \backslash\left(E_{n} \cup F_{n}\right), \quad E_{n+1}=E_{n} \cup F_{n} .
$$

Assume that one starts with $E_{0}=\emptyset$ and some large $F_{0}$. One fundmental question asks whether a "ring of fire" $F_{n}$ can survive forever (i.e. $F_{n} \neq \emptyset$ for all $n$ ). The following theorem provides a partial answer, which, although much better than Corollary 1 in [GG1], is still not best possible.

Theorem 1.5. Assume that the growth dynamics given by $\mathcal{T}$ are convex and that $K_{1 / w}$ is strictly convex. Assume also that there exists a neighborhood $\mathcal{N}$ for $\mathcal{T}$ and an $\eta>0$ such that, for every $u \in S^{1}$,

$$
-\mathcal{N} / 2+B_{2}(0, \eta) \subset L .
$$

Then there exists an $\epsilon>0$ so that for a convex initial set $E_{0}$ with $\mathcal{C}^{2}$ boundary and curvature $<\epsilon$,

$$
\frac{1}{n} F_{n} \rightarrow \partial L
$$

as $n \rightarrow \infty$. In particular, $F_{n} \neq \emptyset$ for every $n$.

As an example when (1.2) is satisfied, consider threshold growth with $\mu$ Lebesgue measure on a compact set $\mathcal{N}$. Let $\theta_{0}=\theta_{0}(\mathcal{N})$ be the largest $\theta$ for which $-\mathcal{N} / 2 \subset$ $L$. Then one can easily check that (1.2) is satisfied as soon as $\theta<\theta_{0}$. The theorem applies, for example, to $\mathcal{N}=B_{p}(0,1), 1<p<\infty ; \theta_{0}\left(B_{p}(0,1)\right)$ equals $\pi / 3-\sqrt{3} / 4 \approx 0.614$ if $p=2$ and converges to 0.5 as $p \rightarrow \infty$. To see how far this is from optimal, we define $\theta_{1}=\theta_{1}(\mathcal{N})$ to be the maximal $\theta$ for which the fire survives started from any half-space $E_{0}=H_{u}^{-}$. It is not hard to see that $\theta_{1}\left(B_{p}(0,1)\right)$ equals $2 \max _{x \in[0,1]} \int_{x}^{2 x} \sqrt{1-u^{2}} d u \approx 0.6435$ if $p=2$, and converges to $2 \max _{x \in[0, \sqrt{2}]} \int_{x}^{2 x}(\sqrt{2}-u) d u=2 / 3$ as $p \rightarrow \infty$. We conjecture (see also [FGG] and [GG1]) that the fire can survive from a bounded $E_{0}$ iff $\theta<\theta_{1}$. We are unable to prove this for the simple reason that we are unable, under reasonable assumptions, 
to show convexity of $E_{n}$ for every $n$. However, for $\theta \in\left(\theta_{0}, \theta_{1}\right)$, the limiting set of $F_{n} / n$ can clearly no longer be $\partial L$.

Unfortunately, our results yield no information in cases when $K_{1 / w}$ fails to be strictly convex, as, for example, it does in the case of threshold growth with $\mu$ Lebesgue measure on $B_{\infty}(0,1)$ ([GG1]), or with $\mu$ a counting measure on a finite subset of $\mathbf{Z}^{2}$ ([GG2]). New and more robust techniques are needed to prove a satisfactory version of (1) in Theorem 1.3 in these cases.

In conclusion, we should mention that the methods used to prove Theorems 1.31.5 extend to higher dimensions, albeit with considerable technical difficulties. However, $K_{1 / w}$ is very rarely convex even in three dimensions, so the two-dimensional case is the most compelling.

\section{Preliminaries}

2.1. Convexity. Convexity properties of threshold growth dynamics were addressed in [GG1], with the following conclusions.

Proposition 2.1. Let $\mathcal{T}$ be a threshold growth transformation with $\mu$ the Lebesgue measure restricted to a compact convex $\mathcal{N} \in \mathcal{B}$.

(1) $\mathcal{T}$ is convex for every $\theta>0$.

(2) If $\mathcal{N}$ is symmetric (i.e. $-\mathcal{N}=\mathcal{N}$ ), then $K_{1 / w}$ is convex.

(3) If $\mathcal{N}$ is strictly convex and symmetric, then $K_{1 / w}$ is strictly convex.

(4) If $\mathcal{N}$ is $\mathcal{C}^{1}$, then $w$ is $\mathcal{C}^{2}$.

Proof. See Propositions A1, A3 and A4 in [GG1].

The next two proposition provide additional examples of convex dynamics.

Proposition 2.2. Let $\mathcal{T}$ define Additive Dynamics with convex $\mathcal{N}$. Then $\mathcal{T}$ is convex, and $K_{1 / w}=-\mathcal{N}^{*}$ is convex. In addition, $\mathcal{N}$ is $\mathcal{C}^{1}$ iff $K_{1 / w}$ is strictly convex.

Proof. These are rephrased standard results.

Proposition 2.3. Let $\mathcal{T}$ be Inclusion Dynamics with compact convex $\mathcal{N}$. Then $\mathcal{T}$ is convex.

Proof. Let $A$ be a closed convex set. Then $\mathcal{T}(A)$ is clearly closed. To see why it is convex, assume that $x_{1}, x_{2} \in \mathcal{T}(A)$, so that there exist $y_{1}, y_{2}$ such that $y_{i}+\mathcal{M} \subset$ $A \cap\left(x_{i}+\mathcal{N}\right), i=1,2$. Pick an $\alpha \in(0,1)$. Then $\alpha y_{1}+(1-\alpha) y_{2}+\mathcal{M} \subset \alpha\left(y_{1}+\mathcal{M}\right)+$ $(1-\alpha)\left(y_{2}+\mathcal{M}\right) \subset A \cap\left(\alpha\left(x_{1}+\mathcal{N}\right)+(1-\alpha)\left(x_{2}+\mathcal{N}\right)\right)=A \cap\left(\alpha x_{1}+(1-\alpha) x_{2}+\mathcal{N}\right)$, so that $\alpha x_{1}+(1-\alpha) x_{2} \in \mathcal{T}(A)$.

Unfortunately, it is rather rare for Inclusion Dynamics to have $K_{1 / w}$ convex. For example, if $\mathcal{N}=B_{\infty}(0,1)$, then $K_{1 / w}$ is convex iff the closed convex hull of $\mathcal{M}$ is a rectangle with sides parallel to the axes.

Diameter Dynamics provide an example which is not convex even in the isotropic case $\mathcal{N}=B_{2}(0,1)$ (when both $K_{1 / w}$ and $L$ are $\ell^{2}$ discs). We leave the verification of this to the reader. 
2.2. Curvature. We start by defining two quantities, in addition to $m_{a}(B)$, which measure curvature of a convex set $B$ :

$$
\begin{aligned}
\omega_{a}(B)=\sup \left\{\left\|\nu_{1}-\nu_{2}\right\|_{2}:\right. & \nu_{1} \text { a normal at } x \in \partial B, \\
& \left.\nu_{2} \text { a normal at } y \in \partial B,\|x-y\|_{2} \leq a\right\}
\end{aligned}
$$

and

$$
\begin{gathered}
\bar{m}_{a}(B)=\inf \{\lambda>0: \text { there exists an } x \in \partial B \text { and a normal } \nu \text { at } x \text { so that } \\
\left.\qquad\left|B_{2}(x-\lambda \nu, a) \cap B\right| \geq \frac{1}{2}\left|B_{2}(0, a)\right|\right\} .
\end{gathered}
$$

Also, if $B$ has $\mathcal{C}^{2}$ boundary, then we denote by $\kappa^{*}(B)=\max \{-\kappa(x): x \in \partial B\}$, where $\kappa(x)$ is the curvature of $\partial B$ at $x$.

Let us now establish some relations between $m, \omega$ and $\kappa$, beginning with the obvious ones:

$$
\begin{aligned}
& \omega_{a}(B) \leq \kappa^{*}(B) \cdot a, \\
& \omega_{2 a}(B) \leq 2 \cdot \omega_{a}(B) .
\end{aligned}
$$

Next, for $B$ with $\omega_{2 a}(B) \leq 1$, we claim that

$$
m_{a}(B) \leq 2 \omega_{2 a}(B) .
$$

To prove $(2.3)$, note that $m_{a}(B) \leq k$ as soon as $\omega_{\sqrt{a^{2}+k^{2} a^{2}}}(B) \leq r$. Here, $r$ is such that $\|u-v\|_{2} \leq r$ implies that $\tan \phi \leq k$, where $\phi$ is the angle between $u$ and $v$. A short computation gives that $\tan \phi \leq 2\|u-v\|$ if $\|u-v\| \leq 1$, proving (2.3).

There is a converse inequality to $(2.3)$,

$$
m_{2 a}(B) \geq \frac{a}{2} \cdot \omega_{a}(B)
$$

which works because (with notation as above), $\tan \phi \geq\|u-v\|_{2} / 2$, so that $\omega_{a}(B) \geq$ $r$ implies that $m_{2 a}(B) \geq \frac{1}{2} r \cdot a$.

Note that (2.2), (2.3) and (2.4) imply that, for our purposes, it is irrelevant which particular $a$ is used in $m_{a}$, and that $m_{a}$ and $\omega_{a}$ are interchangeable.

Lemma 2.4. Let $A$ be a convex set with $\mathcal{C}^{1}$ boundary. There exists a sequence $A_{k}$ of convex sets with $\mathcal{C}^{2}$ boundary so that, for large enough $k, d_{H}\left(A, A_{k}\right) \leq 1 / k$ and

$$
\kappa^{*}\left(A_{k}\right) \leq k \cdot \omega_{1 / k}(A) .
$$

Proof. Let $\mathcal{T}$ define the threshold growth dynamics with $\mathcal{N}=B_{2}(0,1), \mu$ the Lebesgue measure on $\mathcal{N}$, and $\theta<\pi / 2$ but close to $\pi / 2$. We simply define

$$
A_{k}=k^{-1} \mathcal{T}(k A) \text {. }
$$

Then obviously $d_{H}\left(A, A_{k}\right) \leq 1 / k$. Therefore, we only need to estimate $\kappa^{*}\left(A_{k}\right)$.

To do this, let $B$ be a convex set with $\mathcal{C}^{1}$ boundary and small $\omega_{1}(B)$. Fix an $x_{0} \in \partial B$, and translate so that $x_{0}=(0,0)$. In a neighborhood of $x_{0}, \partial B$ is given by the graph of $y=f(x)$, with $f(0)=0$. Let $V(x, \lambda)$ be the volume of $((x, \lambda)+\mathcal{N}) \cap B$. Then $\partial(\mathcal{T}(A))$ is represented by the graph of $y=g(x)$, where $V(x, g(x))=\theta$.

Denote by $\left(\xi_{1}, \eta_{1}\right)$ and $\left(\xi_{2}, \eta_{2}\right)$ the two points in $((x, \lambda)+\mathcal{N}) \cap \partial B$, and $k_{1}, k_{2}$ (resp. $\left.l_{1}, l_{2}\right)$ the slopes of tangents to $\partial B($ resp. $(x, \lambda)+\mathcal{N})$ ) at these points (see 


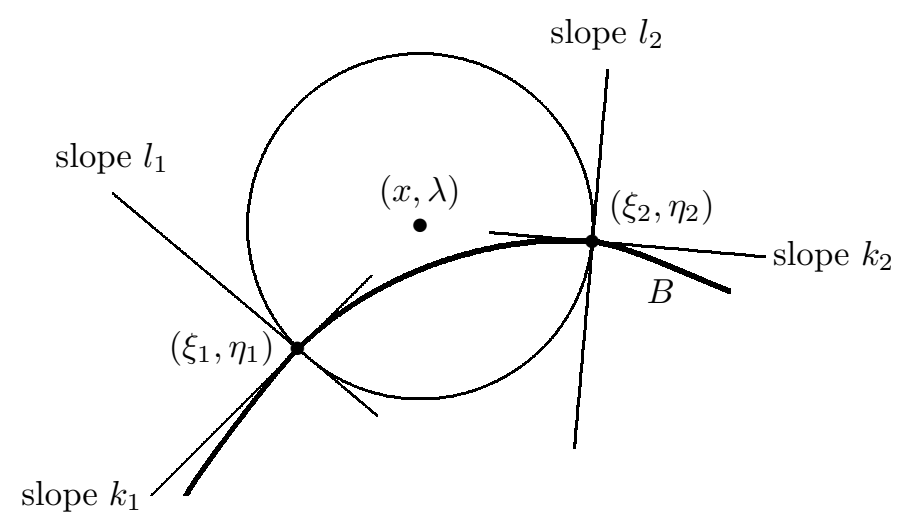

Figure 1. Quantities in (2.5).

Figure 1). Then,

$$
\begin{aligned}
& \frac{\partial V}{\partial \lambda}=-\left(\xi_{2}-\xi_{1}\right), \\
& \frac{\partial V}{\partial x}=\eta_{2}-\eta_{1}, \\
& \frac{\partial^{2} V}{\partial x^{2}}=\frac{k_{2} l_{2}}{l_{2}-k_{2}}-\frac{k_{1} l_{1}}{l_{1}-k_{1}} .
\end{aligned}
$$

By a suitable rotation, we can assume, without loss of generality, that $\partial V / \partial x=$ $\eta_{2}-\eta_{1}=0$ at $x=0$, so that $g^{\prime}(0)=0$ and

$$
g^{\prime \prime}(0)=-\frac{\partial^{2} V}{\partial x^{2}} / \frac{\partial V}{\partial \lambda}=\frac{1}{\xi_{2}-\xi_{1}}\left(k_{2}-k_{1}+\frac{k_{2}^{2}}{l_{2}-k_{2}}-\frac{k_{1}^{2}}{l_{1}-k_{1}}\right) .
$$

Since $k_{1} \geq 0, k_{2} \leq 0, l_{1} \leq 0, l_{2} \geq 0$,

$$
g^{\prime \prime}(0) \geq \frac{k_{2}-k_{1}}{\xi_{2}-\xi_{1}} .
$$

Since $\xi_{2}-\xi_{1}$ must be greater than 1 if $k$ is large enough, and $k_{2}-k_{1} \approx\left\|\nu_{2}-\nu_{1}\right\|$ (where $\nu_{1}$ and $\nu_{2}$ are normals at the respective points), it follows that $\kappa^{*}\left(A_{k}\right)=$ $k \cdot \kappa^{*}(\mathcal{T}(k A)) \leq k \cdot \omega_{1}(k A)=k \cdot \omega_{1 / k}(A)$.

We should remark that the above proof shows that the isotropic threshold growth transformation decreases curvature of convex sets. This is not in general the case for other threshold growth dynamics, since the inequalities $l_{1} \leq 0, l_{2} \geq 0$ may fail to hold.

Lemma 2.5. There exists an $\epsilon>0$ so that if $A$ and $B$ are closed convex sets and $\omega_{1}(A)<\epsilon$, then

$$
d_{H}(\mathcal{T}(A), \mathcal{T}(B)) \leq d_{H}(A, B) \cdot\left(1+\omega_{8 R}(A)^{2}\right) .
$$

Proof. Assume that $d_{H}(A, B)=r$. Write

$$
\begin{aligned}
m_{4 R}^{\prime}(A)=\max \left\{1 /\left\langle\nu_{1}, \nu_{2}\right\rangle:\right. & \nu_{1} \text { normal at } x \\
& \in \partial A, \\
\nu_{2} \text { normal at } y & \left.\in \partial A,\|x-y\|_{2} \leq 4 R\right\} .
\end{aligned}
$$


Then it is easy to see that, for any $u \in S^{1}$ and $r, \lambda>0, \lambda u \in \mathcal{T}\left(A+B_{2}(0, r)\right)$ implies $\lambda u \in \mathcal{T}\left(A+r m_{4 R}^{\prime}(A) u\right)=\mathcal{T}(A)+r m_{4 R}^{\prime}(A) u$. Therefore,

$$
\mathcal{T}(B) \subset \mathcal{T}\left(A+B_{2}(0, r)\right) \subset \mathcal{T}(A)+B_{2}\left(0, r m_{4 R}^{\prime}(A)\right) .
$$

On the other hand, if we define $A \ominus B_{2}(0, r)=\left(A^{c}+B_{2}(0, r)\right)^{c}$, then $m_{4 R}^{\prime}\left(A \ominus B_{2}(0, r)\right) \leq m_{8 R}(A)$ and hence

$$
\mathcal{T}(A) \ominus B_{2}\left(0, r m_{8 R}^{\prime}(A)\right) \subset \mathcal{T}\left(A \ominus B_{2}(0, r)\right) \subset \mathcal{T}(B) .
$$

Therefore, we only need to estimate $m_{8 R}^{\prime}(A)$. However, using $\left\langle\nu_{1}, \nu_{2}\right\rangle=1-$ $\frac{1}{2}\left\|\nu_{1}-\nu_{2}\right\|_{2}^{2}$, one easily arrives at (2.6).

Lemma 2.6. If $A$ and $B$ are convex sets with $\mathcal{C}^{2}$ boundary, then $\kappa_{A+B}^{*} \leq$ $\left(1 / \kappa_{A}^{*}+1 / \kappa_{B}^{*}\right)^{-1}$.

Proof. Curvature is the reciprocal radius of the second order fit circle.

Lemma 2.7. There exists an $\epsilon>0$ so that if $A$ and $B$ are two convex sets with $\omega_{1}(A)<\epsilon, \omega_{1}(A) \cdot d_{H}(A, B)<\epsilon$, and $d_{H}(A, B) \geq 1000 \omega_{1}(A)$, then

$$
\omega_{1}(B) \leq 25 \sqrt{\omega_{1}(A) \cdot d_{H}(A, B)} .
$$

Proof. Let $\alpha=\omega_{1}(A), \beta \leq \omega_{1}(B)$ and $h=d_{H}(A, B)$. Assume that that $\partial A$ goes through $(0,0)$ and that $-e_{2}$ is the normal to $\partial A$ at the origin. Then it is easy to see that $\partial A$ must be below $y=2 \alpha x^{2}$ for $x \in[-\delta / \alpha, \delta / \alpha]$ as soon $\delta$ is small enough.

Moreover, we can assume that $B$ is entirely above the lines $y=-h-\frac{\beta}{4}(x+1)$, $y=-h, y=-h+\frac{\beta}{4}(x-1)$. Therefore, the point $\left(x_{0}, y_{0}\right)$ with $x_{0}=\beta /(16 \alpha)$ and $y_{0}=2 \alpha x_{0}^{2}$ is, for $\beta$ small enough, at least $\frac{1}{2}\left(\beta^{2} /(128 \alpha)-\beta / 4-h\right)$ away from $B$, hence the point on $\partial A$ with the same $x_{0}$ cannot be closer to $A$.

It follows that $\beta^{2} /(128 \alpha)<\beta+3 h$. Since we can assume that $\beta \leq h$ (otherwise the claim follows immediately), we get that $\beta \leq \sqrt{512 \cdot h \alpha}$, ending the proof.

Lemma 2.8. Assume that $\mathcal{T}$ is supercritical, and that $K_{1 / w}$ is convex. There exists a constant $C$ (depending only on $\mathcal{T}$ ) and an $\epsilon>0$ so that for every $\mathcal{C}^{2}$ convex set $A$ with $\kappa^{*}(A)<\epsilon, d_{H}(\mathcal{T}(A), A+L)<C \cdot \kappa^{*}(A)$.

Proof. Note that $K_{1 / w}$ convex is equivalent to $H_{u}^{-}+L=H_{u}^{-}+w(u) u$ for every $u \in S^{1}$. Then, by (2.1) and (2.3), there exists a $C$ so that $A+\left(1-C \cdot \kappa^{*}(A)\right) L \subset$ $\mathcal{T}(A) \subset A+L$.

We end this section with a result that gives a simple lower bound on $\bar{m}$. Let $\bar{\omega}_{a}(A)=\min \left\{\left\|\nu_{1}-\nu_{2}\right\|_{2}: \nu_{1}\right.$ and $\nu_{2}$ are normals of boundary points at distance $\geq a\})$.

Lemma 2.9. Assume $A$ is a strictly convex set with $\mathcal{C}^{1}$ boundary. Then $\bar{m}_{a}(A) \geq$ $0.1 \cdot a^{2} \cdot \bar{\omega}_{\eta}(A)$, for small enough $\eta$.

Proof. Let $\delta=\bar{\omega}_{\eta}(A)$. In local coordinates, $\partial A$ is given by a concave function $y=f(x)$ with $f^{\prime}(0)=0$. If $\eta$ is small enough, $f^{\prime}(x) \leq-0.9 \delta x$, so that $f(x) \leq$ $-0.9 \delta x^{2} / 2$, and thus $\bar{m}_{a}(A)$ is at least $\epsilon$ given by the equation $\int_{-a}^{a}\left(-0.9 \delta x^{2}+\epsilon\right) d x=$ 0 , giving the specified bound. 


\section{Proofs of Theorems $1.3-1.5$}

Lemma 3.1. Assume that a sequence of positive numbers $p_{k}$ satisfies $p_{k+1} \leq$ $p_{k} /\left(1+f\left(p_{k}\right) p_{k}^{3 / 2}\right)$, where $f$ is a positive decreasing function such that $\lim _{p \rightarrow 0} f(p)=$ $\infty$. Then $\lim _{k \rightarrow \infty} p_{k} k^{2 / 3}=0$. Moreover, for every $\epsilon>0$ there exists a $\delta>0$ so that $p_{1}<\delta$ implies $\sup _{k} k^{2 / 3} p_{k}<\epsilon$.

Proof. It is clear that $p_{k}$ must go to 0 . Fix a large $M$ and let $k_{0}$ be large enough so that $f\left(p_{k}\right) \geq M$ and $p_{k} \leq(M / 2)^{-2 / 3}$ for $k \geq k_{0}$. Then $p_{k+1} \leq p_{k} /\left(1+M p_{k}^{3 / 2}\right)$ for $k \geq k_{0}$. We claim that this implies that $p_{k} \leq\left(M\left(k-k_{0}+1\right) / 2\right)^{-2 / 3}$, which clearly finishes the proof.

The claim can be proved by induction. Before checking the induction step, note that $g(x)=x /\left(1+M x^{3 / 2}\right)$ is increasing on $\left[0,(M / 2)^{-2 / 3}\right]$. Therefore, the induction step reduces to showing that $g\left(\left(M\left(k-k_{0}+1\right) / 2\right)^{-2 / 3}\right) \leq\left(M\left(k-k_{0}+2\right) / 2\right)^{-2 / 3}$ for every $k \geq k_{0}$. Clearly it is enough to check this for $k_{0}=1$, in which case it reduces to $(1+1 / k)^{2 / 3} \leq 1+2 / k$. This also proves the claim in the last sentence of the statement.

In the proofs below, $C$ is a generic constant, changing value from one appearance to another.

Proof of Theorem 1.3. For a given small $\epsilon>0$, choose a set $L(\epsilon)$ so that $d_{H}(L, L(\epsilon))$ $\leq \sqrt{\epsilon}$ and $\kappa^{*}(L(\epsilon)) \leq \omega_{1}(L, \sqrt{\epsilon}) / \sqrt{\epsilon}$. Note that the existence of $L(\epsilon)$ is guaranteed by Lemma 2.4 together with the fact that $L$ has $\mathcal{C}^{1}$ boundary since $K_{1 / w}$ is strictly convex.

Choose the sets $B_{1}, B_{2}, \ldots, L_{1}, L_{2}, \ldots$, and small numbers $p_{1}, p_{2}, \ldots$ in the following fashion. Let $B$ be a $\mathcal{C}^{2}$ convex set with $\kappa^{*}(B)$ small and

$$
\begin{aligned}
& p_{1}=\kappa^{*}(B), \quad L_{1}=L\left(p_{1}\right), \quad B_{1}=B+L_{1}, \\
& p_{2}=\kappa^{*}\left(B_{1}\right), \quad L_{2}=L\left(p_{2}\right), \quad B_{2}=B_{1}+L_{2}, \\
& p_{3}=\kappa^{*}\left(B_{2}\right), \quad L_{3}=L\left(p_{3}\right), \quad B_{3}=B_{2}+L_{3}, \\
& \text {... }
\end{aligned}
$$

From Lemma 2.6 it follows that

$$
p_{n+1} \leq \frac{p_{n}}{1+\omega_{1}\left(L, \sqrt{p}_{n}\right)^{-1} p_{n}^{3 / 2}},
$$

and therefore, by Lemma 3.1, $p_{n}=n^{-3 / 2} \epsilon_{n}$ with $\lim _{n \rightarrow \infty} \epsilon_{n}=0$. Moreover, also by Lemma 3.1, if $\kappa^{*}(B)$ is small enough, then $\sup _{n} \epsilon_{n}<\epsilon$. Also, by Lemma 2.5 and Lemma 2.8,

$$
\begin{aligned}
d_{H}\left(B_{n}, \mathcal{T}^{n}(B)\right) & \leq d_{H}\left(B_{n}, \mathcal{T}\left(B_{n-1}\right)\right)+d_{H}\left(\mathcal{T}\left(B_{n-1}\right), \mathcal{T}\left(\mathcal{T}^{n-1}(B)\right)\right) \\
& \leq C \sqrt{p}_{n}+\left(1+C p_{n}^{2}\right) \cdot d_{H}\left(B_{n-1}, \mathcal{T}^{n-1}(B)\right),
\end{aligned}
$$

and therefore

$$
d_{H}\left(B_{n}, \mathcal{T}^{n}(B)\right) \leq \prod_{k=1}^{n}\left(1+C p_{k}^{2}\right)\left(\sqrt{p}_{1}+\cdots+\sqrt{p_{n}}\right) \leq C n^{2 / 3} .
$$

To prove (1), use Lemma 2.7 and (2.1)-(2.4) to get

$$
m_{a}\left(\mathcal{T}^{n}(B)\right) \leq C \sqrt{p_{n+1} n^{2 / 3}} \leq C \sqrt{\epsilon}
$$


Since $A_{0}$ generates regular growth, we can choose $r$ and $n_{0}$ large enough so that $A_{0} \subset B \subset \mathcal{T}^{n_{0}}\left(A_{0}\right)$, and we also have $d_{H}\left(B_{n}, \mathcal{T}^{n}\left(A_{0}\right)\right) \leq C n^{2 / 3}$. It now follows from Lemma 2.7 and (2.1)-(2.4) that

$$
m_{a}\left(\mathcal{T}^{n}\left(A_{0}\right)\right) \leq C \sqrt{p_{n+1} n^{2 / 3}} \leq C \sqrt{\epsilon_{n}},
$$

for large $n$, which completes the proof of (2).

Proof of Theorem 1.4. Note that $L$ is $\mathcal{C}^{2}$ and strictly convex in this case. Clearly $\kappa^{*}(n L) \leq C / n$. Therefore, by Lemma $2.8,(n+1-C / n) L \subset \mathcal{T}(n L)$.

Now pick $r$ large and $n_{0}$ even larger so that $r L \subset \mathcal{T}^{n_{0}}\left(A_{0}\right)$. Then $\mathcal{T}^{n}\left(A_{0}\right) \supset$ $\mathcal{T}^{n-n_{0}}(r L) \supset\left(n+r-n_{0}-C \log n\right)$.

Under the conditions of Theorem 1.4, $\mathcal{T}^{n}\left(A_{0}\right)$ is of order $\log n$ away from $n L$ for dynamics which "feel the curvature," as the next proposition shows. We omit the simple proof.

Proposition 3.2. Assume all the hypotheses of Theorem 1.4. Assume also that there exists a constant $D>0$ so that for large $n, \mathcal{T}(n L) \subset(n+1-D / n) L$. Then there exists a constant $C>0$ so that $\mathcal{T}^{n}\left(A_{0}\right) \subset(n-C \log n) L$.

Note that, by Lemma 2.9 and Proposition 2.1, supercritical threshold growth dynamics with strictly convex and symmetric $\mathcal{N}$ with $\mathcal{C}^{1}$ boundary satisfy the hypotheses of this proposition.

At this point let us make a few remarks about Additive Dynamics with convex $\mathcal{N}$. Note first that this is a case where Proposition 3.2 fails. Also, part (1) of Theorem 1.3 always holds (although $K_{1 / w}$ is not always strictly convex), whereas it is easy to see that part (2) does not hold unless $K_{1 / w}$ is strictly convex. This shows that strict convexity is necessary for at least part (2) of Theorem 1.3.

Proof of Theorem 1.5. Condition (1.2) is equivalent to the existence of (a possibly different) $\eta>0$ such that for each $u \in S^{1}$,

$$
(w(u) u+\mathcal{N}) \cap\left(H_{u}^{-}-(w(u)-\eta) u\right)=\emptyset .
$$

This implies that if the curvature of a convex set $A$ is small enough, and $B_{1}=\mathcal{T}(A)$, $B_{2}=\mathcal{T}(A) \backslash A$, then $\mathcal{T}\left(B_{1}\right) \backslash B_{1}=\mathcal{T}\left(B_{2}\right) \backslash B_{1}$. By an inductive argument, it then follows that

$$
\mathcal{T}^{n}\left(E_{0}\right)=E_{n} \cup F_{n},
$$

so that Theorem $1.3(1)$ ends the proof.

\section{ACKNOWLEDGEMENTS}

The author wishes to express his gratitude to David Griffeath for introducing him to the subject of excitable media and many helpful insights. He is also thankful to the referee for a thorough reading of the manuscript.

\section{REFERENCES}

[Boh] T. Bohman, work in preparation.

[DG] R. Durrett, D. Griffeath, Asymptotic behavior of excitable cellular automata, Experimental Math. 2 (1993), 183-208. MR 95e:58095

[Dur] R. Durrett, Lecture Notes on Particle Systems and Percolation, Wadsworth\&Brooks/ Cole, 1988. MR 89k:60157 
[FGG] R. Fisch, J. Gravner, D. Griffeath, Threshold-range scaling for the excitable cellular automata, Statistic and Computing 1 (1991), 23-39.

[GG1] J. Gravner, D. Griffeath, Threshold growth dynamics, Trans. Amer. Math. Soc. 340 (1993), 837-870. MR 94b:52006

[GG2] J. Gravner, D. Griffeath, First passage times for discrete threshold growth dynamics, submitted to Ann. Prob. (1995).

[GG3] J. Gravner, D. Griffeath, Multitype threshold voter model and convergence to PoissonVoronoi tessellation, preprint (1995).

[Gra] J. Gravner, Abstract growth dynamics, unpublished manuscript (1992).

[Gri] D. Griffeath, Self-organization of random cellular automata: four snapshots, Probability and Phase Transition (G. Grimmett, ed.), Kluwer, 1994. MR 95b:82051

[NP] C. M. Newman, M. S. T. Piza, Divergence of shape fluctuations in two dimensions, Ann. Prob. 23 (1995), 977-1005. CMP 95:17

[Pir] G. E. Pires, Threshold Growth Dynamics: a PDE Approach, Ph. D. Thesis, University of Wisconsin, Madison, 1995.

Department of Mathematics, University of California, Davis, California 95616

E-mail address: gravner@feller.ucdavis.edu 